\title{
A 5Gb/s 7.1fJ/b/mm 8× Multi-Drop On-Chip 10mm Data Link in 14nm FinFET CMOS SOI at $0.5 \mathrm{~V}$
}

\author{
Elisa Sacco ${ }^{1,2}$, Pier Andrea Francese ${ }^{1}$, Matthias Brändli ${ }^{1}$, Christian Menolfi ${ }^{1}$, Thomas Morf $^{1}$, \\ Alessandro Cevrero ${ }^{1}$, Ilter Ozkaya ${ }^{1}$, Marcel Kossel ${ }^{1}$, Lukas Kull ${ }^{1}$, Danny Luu ${ }^{1}$, Hazar Yueksel ${ }^{1}$ \\ Georges Gielen ${ }^{2}$ and Thomas Toifl ${ }^{1}$ \\ ${ }^{1}$ IBM Research, Zurich, Switzerland, \\ ${ }^{2}$ KU Leuven - ESAT MICAS, Belgium
}

\begin{abstract}
We report a $5 \mathrm{~Gb} / \mathrm{s}$ data link implemented in $14 \mathrm{~nm}$ FinFET CMOS SOI technology in which a single transmitter (TX) broadcasts NRZ data to eight receivers (RXs) distributed along an on-chip RC-dominated 10mm-long channel. The TX comprises a full-rate AC-coupled 2-tap FIR driver with a quarter-rate pre-driver and aligner. Each RX is equipped with a novel decision-gated 1-tap speculative DFE optimized for low-power. The RX architecture is half-rate and sliced data are de-multiplexed and aligned at quarter-rate so that all the bus interfaces are at $1.25 \mathrm{~Gb} / \mathrm{s}$. PRBS generator and checker are available on-chip. Correct operation was verified with PRBS31 data transmitted at $5 \mathrm{~Gb} / \mathrm{s}$ and concurrently received error-free at each drop with $>40 \%$ horizontal margin $\left(\mathrm{BER}<10^{-12}\right.$ ) at the $\mathrm{RX}$ connected at the end of the channel. At this data-rate the efficiency is $7.1 \mathrm{fJ} / \mathrm{b} / \mathrm{mm}$, resulting in the best performance among multi-drop on-chip data links so far published (to the best of our knowledge). The TX and eight RXs are running on a $0.5 \mathrm{~V}$ power supply and consume 0.62 and $0.98 \mathrm{~mW}$, respectively.
\end{abstract}

\section{Introduction}

Neuromorphic systems, where one artificial neuron communicates with many neurons through synapses, and data-centric systems, where microprocessors and parallel accelerators concurrently operate on the same centrally distributed data, require parallel processing of the same data. These applications drive the interest in the energy-efficient implementation of multi-drop on-chip data links built with wires interconnecting digital blocks placed a few mm apart.

\section{On-Chip Interconnect Channel}

In this work, we used a $10 \mathrm{~mm}$-long differential channel built with $0.5 \mu \mathrm{m}$ thick wires routed through a $5.12 \times 5.12 \mu \mathrm{m}^{2}$ power mesh acting as a shield. The channel was modeled in HFSS. The attenuation is $1.8 \mathrm{~dB} / \mathrm{mm}$ at $2.5 \mathrm{GHz}$ and is RC-dominated with $\mathrm{R}=37 \Omega / \mathrm{mm}$ and $\mathrm{C}=340 \mathrm{fF} / \mathrm{mm}$ at $25^{\circ} \mathrm{C}$. Figure 1 shows the channel cross section, its insertion loss and the layout placement of the circuits connected to it.

\section{AC-Coupled TX}

The TX employs AC coupling, which intrinsically provides pre-emphasis, with selectable unit metal capacitors of $10 \mathrm{fF}$. The additional FFE tap, in which the signal is inverted and delayed one UI, further extends the data rate. In Fig. 2 the implementation is shown together with the quarter-rate pre-driver and timing diagrams. Both the signal amplitude and the equalization strength can be controlled separately by means of 32 units allocated to the cursor and 16 to the post-cursor. Figure 3 presents the eye diagram traces at two points along the channel with the TX FFE turned off and on. Our equalization strategy is to select the best TX FFE setting for the farthest RX and use the DFE at each RX to refine the sensitivity.

Multi-Drop RX

In a multi-drop link the cumulative loading of all the connected RXs is of concern. With AC coupling each RX should present a sufficiently high differential input impedance $Z_{\text {diff }}$ to minimize DC baseline wandering and at the same time a sufficiently low common-mode $Z_{c m}$ to ensure a short start-up settling time to the desired $V_{c m}$ (2/3 of $V_{d d}$ in our design).

The circuit shown in Fig. 4 is very effective in achieving our goal. In common mode the power rail is equally portioned by the three stacked diode-connected NMOS transistors. In differential mode the cross-coupled NMOS transistors generate a negative transconductance $g_{m}$ that cancels the $g_{m}$ of the NMOS diodes above them, thus setting a high $Z_{\text {diff }}$ equal to their $r_{o}$. The small difference between the PRBS7 and PRBS31 horizontal margins of the measured BER bathtubs down to $10^{-12}$ (Fig. 5) confirms the correct operation of the adopted circuit.

A benefit of a multi-drop system is that the TX power is amortized over the number of served RXs. The efficiency $\eta$ is calculated as:

$$
\eta=\frac{P_{T X}+n_{\text {bits }} \cdot P_{R X}}{f_{T X} \cdot L_{\text {chan }} \cdot n_{\text {bits }}}[\mathrm{J} / \mathrm{b} / \mathrm{mm}]
$$

It saturates with increasing number of bits $n_{b i t s}$ concurrently received, i.e. eight in our implementation. $L_{\text {chan }}$ is the average channel length equal to $5.625 \mathrm{~mm} . P_{T X}$ and $P_{R X}$ are the average power consumed by the TX and RX. $f_{T X}$ is the transmission rate.

\section{Decision-Gated DFE}

Each RX operates at half-rate and features a novel low-power 1-tap speculative DFE architecture that reduces the power consumption and the kick-back effect by powering up only the speculative path that is going to take the next decision based on the previous decision. Compared to the usual speculative DFE implementation [1], power is saved because the path not selected by the DFE multiplexer is kept in reset state by gating its clock with the decision taken in the previous UI. As an example, Fig. 6 shows the operation when the even slice detects the current bit while the odd slice is holding a logic one as the previously detected bit.

The slicers are low-noise two-stage dynamic comparators in which the second regenerating stage is self-timed and shared between the first stages of the two speculative comparators to further reduce power. The threshold voltages are generated with 8-bit monotonic resistor-ladder DACs built with highly ohmic and compact poly stripes and switches connected from $(2 / 3-1 / 6)$ to $(2 / 3+1 / 6)$ of the supply rail. The measured input-referred noise of the comparators is $3.1 \mathrm{mV}_{\text {rms. }}$.

The DFE equalization is used in each RX to increase the vertical BER margin in both cases when the signal is under- or over-equalized. The optimal TX FFE setting chosen at the end of the channel must be shared among all the other RXs. The RXs closer to the TX will then be over-equalized as already shown in Fig. 3. However, thanks to our DFE negative range, their vertical margin can be restored. For example, in the BER contour plots (time vs. DFE H1 level) in Fig. 3, the vertical 
margin at BER $10^{-8}$ improves from 18 to $31 \mathrm{mV}_{\mathrm{ppd}}$ in the last RX located at the end of the channel, and from 38 to $>60 \mathrm{mV}_{\mathrm{ppd}}$ in the RX connected $2.5 \mathrm{~mm}$ away from the TX.

\section{Measurement Setup}

The prototype circuit was measured with wafer needle probing. The BER tests are performed with an on-chip PRBS generator and checker physically placed below the channel and interfaced at quarter-rate with the TX pre-driver and each of the RX de-multiplexer/aligner. The BER bathtubs horizontal openings are shown in Fig. 5 together with the delay of the bathtub centers with respect to the input CLK. The measured latency is $21 \mathrm{ps} / \mathrm{mm}$.

\section{Conclusion}

This work is significantly better than the previously published results of on-chip multi-drop data links [2, 3] with respect to both the reported efficiency, which is more than eight times better, and the channel dimensions, i.e., length and pitch density. Moreover, two point-to-point links $[4,5]$ are included in the comparison table shown in Fig. 7. In that case, the comparison is made with only the RX at the end of our channel turned on, thus $n_{\text {bits }}$ and $L_{\text {chan }}$ are equal to 1 and $10 \mathrm{~mm}$, respectively. We underline that $P_{T X}$ and $P_{R X}$ reported in this work also include clock generation, serializer, de-serializer and voltage reference generation.

\section{References}

[1] D. Turker et al., "A 19Gb/s 38mW 1-Tap Speculative DFE Receiver in 90nm CMOS," pp. 216-217, VLSI 2009.

[2] H. Ito et al., "A 8-Gbps Low-Latency Multi-Drop On-Chip Transmission Line Interconnect with $1.2-\mathrm{mW}$ Two-Way Transceivers," pp. 136-137, VLSI 2007.

[3] H. Wu et al., "A $60 \mathrm{GHz}$ On-Chip RF-Interconnect with $\lambda / 4$ Coupler for $5 \mathrm{Gbps}$ Bi-Directional Communication and Multi-Drop Arbitration," CICC 2012.

[4] S. Lee et al., "A $95 \mathrm{fJ} / \mathrm{b}$ current-mode transceiver for $10 \mathrm{~mm}$ onchip interconnect," pp. 262-263, ISSCC 2013.

[5] Y. Liu et al., "A $0.1 \mathrm{pJ} / \mathrm{b}$ 5-to-10 Gb/s charge-recycling stacked low power I/O for on-chip signaling in $45 \mathrm{~nm}$ CMOS SOI," pp. 400-401, ISSCC 2013.


Fig. 1. On-chip channel and test circuit implementation overview.

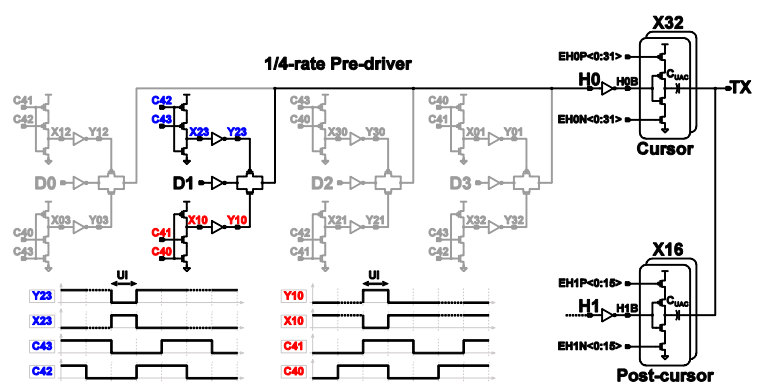

Fig. 2. TX schematic and timing diagram.
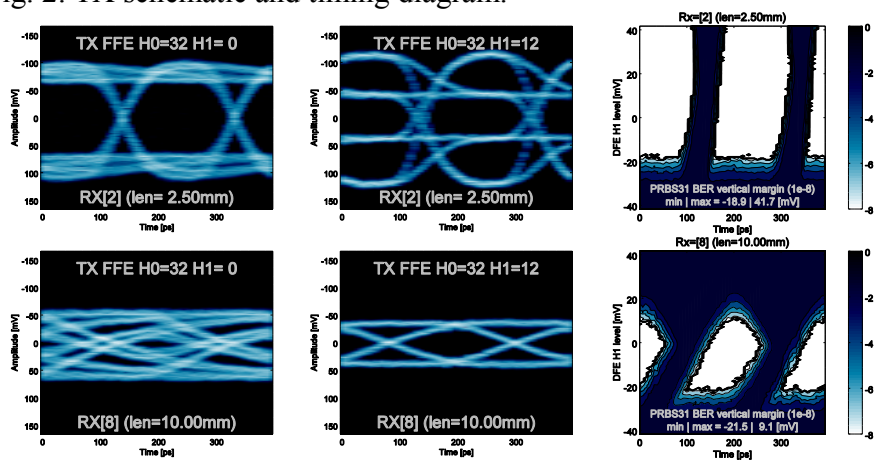

Fig. 3. Eye diagrams FFE off/on and BER vs. H1 at 2.5 and $10 \mathrm{~mm}$.



Fig. 4. Circuits setting CM voltage at the RX inputs.
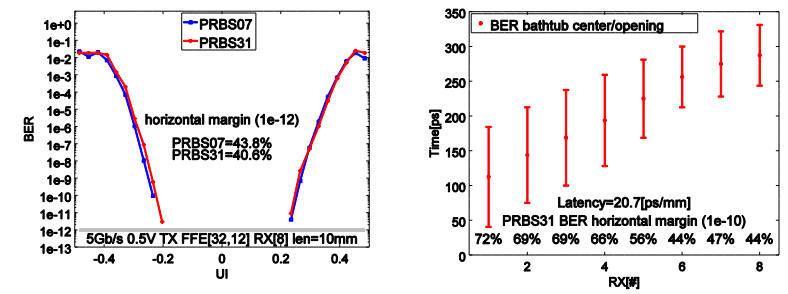

Fig. 5. BER bathtub at the farthest RX and center/opening vs. RX.

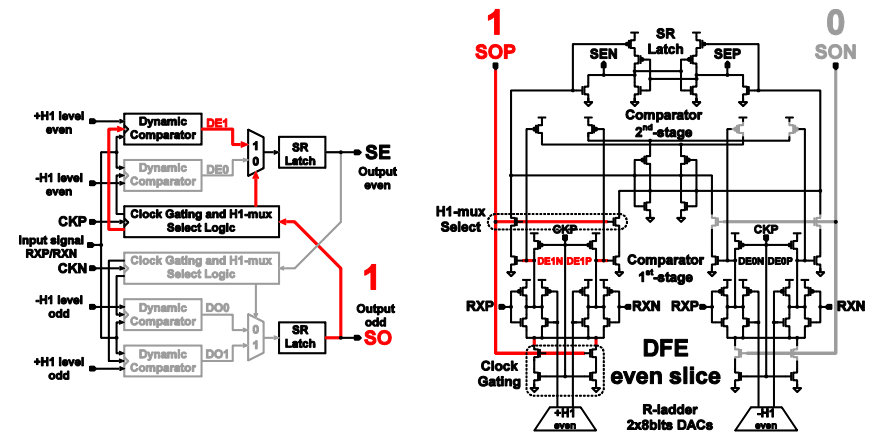

Fig. 6. Decision-gated DFE block diagram and circuit implementation.

\begin{tabular}{|c|c|c|c|c|c|c|}
\hline & & [2] & [3] & [4] & [5] & this work \\
\hline \multicolumn{2}{|c|}{ Technology [nm] } & 90 & 65 & 65 & 45 & 14 \\
\hline \multicolumn{2}{|c|}{ Supply [V] } & 1 & na & 0.9 & 0.9 & 0.5 \\
\hline \multicolumn{2}{|c|}{ Transmission rate $[\mathrm{Gb} / \mathrm{s}]$} & 8 & 5 & 3 & 5 & 5 \\
\hline \multicolumn{2}{|c|}{ BER |PRBS } & $10^{-12} \mid$ na & $10^{-12} \mid 15$ & $10^{-12} \mid \mathrm{na}$ & $10^{-10} \mid 7$ & $10^{-12} \mid 31$ \\
\hline \multicolumn{2}{|c|}{ Channel length $[\mathrm{mm}] \mid$ pitch $[\mu \mathrm{m}]$} & $5 \mid 20$ & $5.5 \mid \mathrm{na}$ & $10 \mid 5$ & $6.5 \mid 4$ & $10 \mid 5.12$ \\
\hline \multicolumn{2}{|c|}{ Multi-drop nbit [\#] } & 6 & 4 & 1 & 1 & 8 \\
\hline \multirow{2}{*}{$\begin{array}{l}\text { Efficiency } \\
{[\mathrm{fJ} / \mathrm{b} / \mathrm{mm}]}\end{array}$} & nbit $=\#$ & 59 & 120 & - & - & $7.1^{*}$ \\
\hline & nbit $=1$ & - & - & 9.5 & 18 & $14.8^{*}$ \\
\hline
\end{tabular}

${ }^{*}$ Clock generation, Serializer, De-serializer and Voltage References Included

Fig. 7. Comparison table of on-chip interconnect data links. 